\title{
Comportamento de nidificação de Melipona subnitida (Ducke, 1910) e Frieseomelitta sp. no Seridó oriental do Rio Grande do Norte, Brasil
}

Nesting behavior of Melipona subnitida (Ducke, 1910) and Frieseomelitta sp. in the eastern Seridó of Rio Grande do Norte, Brazil

Comportamiento de anidación de Melipona subnitida (Ducke, 1910) y Frieseomelitta sp. en el Seridó oriental de Rio Grande do Norte, Brasil

\section{Resumo}

Abelhas indígenas são importantes polinizadores de plantas nativas. Na Caatinga existem cerca de 193 espécies de abelhas pertencentes a 79 gêneros, dos quais 18 são meliponínios. Objetivou-se neste trabalho verificar se existe influência dos pontos cardeais e colaterais na nidificação de abelhas Melipona subnitida Ducke e Frieseomelitta sp. em hospedeiros vegetais da Caatinga. O trabalho foi desenvolvido em Santana do Seridó, estado do Rio Grande do Norte, na Fazenda Morada da Jandaíra $\left(6^{\circ} 44^{\prime} 16^{\prime \prime} \mathrm{S}\right.$ e $\left.36^{\circ} 43^{\prime} 13^{\prime} \mathrm{W}\right)$. As medidas de largura e altura dos ninhos foram feitas com o auxílio de uma fita métrica. Utilizou-se um aplicativo de bússola digital para smartphone (iPhone ${ }^{\circledR}$ Model A 1533) para a leitura do posicionamento dos orifícios de nidificação em relação aos pontos cardeais e colaterais. A velocidade do vento, umidade e temperatura foi realizada por uma mini-estação meteorológica Oregon Scientific ${ }^{\circledR}$, WMR928NX. As análises de frequência absoluta foram feitas no software R v. 2.15 (R Development Core Team, 2005). Foram analisados um total de 136 ninhos de abelhas. Observou-se que as abelhas M. subnitida Ducke e Frieseomelitta sp. nidificam com maior frequência em Commiphora leptophloeos e Poincianella pyramidalis. Quanto a orientação magnética de nidificação, essas abelhas possuem hábitos distintos: a abelha $M$. subnitida Ducke demonstra maior preferência de nidificação na direção Noroeste (NW), enquanto que a abelha Frieseomelitta sp. possui uma preferência de nidificação abrangente, em qualquer orientação magnética, exceto entre os ângulos 270 e $315^{\circ}$.

Palavras-chave: Caatinga; Meliponicultura; Nidificação; Melipona subnitida Ducke; Frieseomelitta sp.

\section{Abstract}

Indigenous bees are important pollinators of native plants. In the Caatinga there are about 193 species of bees belonging to 79 genera, of which 18 are meliponis. The objective of this work was to verify if there is influence of cardinal and collateral points in the nesting of bees Melipona subnitida Ducke and Frieseomelitta sp. in plant hosts from Caatinga. The work was developed in Santana do Seridó, state of Rio Grande do Norte, at Fazenda Morada da Jandaíra $\left(6^{\circ} 44^{\prime}\right.$ 
$16^{\prime \prime} \mathrm{S}$ and $\left.36^{\circ} 43^{\prime} 13^{\prime \prime} \mathrm{W}\right)$. The width and height measurements of the nests were made with the aid of a measuring tape. A digital compass application for smartphone (iPhone ${ }^{\circledR}$ Model A 1533) was used to read the positioning of the nesting holes in relation to cardinal and collateral points. Wind speed, humidity and temperature were measured by an Oregon Scientific ${ }^{\circledR}$ mini meteorological station, WMR928NX. Absolute frequency analyzes were performed using the R v software. 2.15 (R Development Core Team, 2005). A total of 136 bee nests were analyzed. It was observed that the bees M. subnitida Ducke and Frieseomelitta sp. nest more frequently in Commiphora leptophloeos and Poincianella pyramidalis. As for the magnetic nesting orientation, these bees have distinct habits: the M. subnitida Ducke bee demonstrates greater nesting preference in the Northwest (NW) direction, while the Frieseomelitta sp. bee has a broad nesting preference in any magnetic orientation, except between 270 and $315^{\circ}$ angles.

Keywords: Caatinga; Meliponiculture; Nesting; Melipona subnitida Ducke; Frieseomelitta sp.

\begin{abstract}
Resumen
Las abejas indígenas son importantes polinizadores de plantas nativas. En la Caatinga hay alrededor de 193 especies de abejas pertenecientes a 79 géneros, de los cuales 18 son meliponis. El objetivo de este trabajo fue verificar si existe influencia de puntos cardinales y colaterales en la anidación de abejas Melipona subnitida Ducke y Frieseomelitta sp. en plantas hospedantes de Caatinga. La obra se desarrolló en Santana do Seridó, estado de Rio Grande do Norte, en la Fazenda Morada da Jandaíra (6 ${ }^{\circ} 44^{\prime} 16^{\prime \prime} \mathrm{S}$ y $\left.36^{\circ} 43^{\prime} 13^{\prime} \mathrm{W}\right)$. Las medidas de ancho y alto de los nidos se realizaron con la ayuda de una cinta métrica. Se utilizó una aplicación de brújula digital para teléfono inteligente (iPhone ${ }^{\circledR}$ Modelo A 1533) para leer el posicionamiento de los orificios de anidación en relación con los puntos cardinales y colaterales. La velocidad del viento, la humedad y la temperatura se midieron con una mini estación meteorológica de Oregon Scientific $^{\circledR}$, WMR928NX. Los análisis de frecuencia absoluta se realizaron utilizando el software R v. 2.15 (Equipo central de desarrollo de R, 2011). Se analizaron un total de 136 nidos de abejas. Se observó que las abejas M. subnitida Ducke y Frieseomelitta sp. anidan con mayor frecuencia en Commiphora leptophloeos y Poincianella pyramidalis. En cuanto a la orientación de anidación magnética, estas abejas tienen hábitos distintos: la abeja M. subnitida Ducke demuestra una mayor preferencia de anidación en la dirección Noroeste (NW), mientras que la abeja Frieseomelitta sp. tiene una amplia preferencia de anidación en cualquier orientación magnética, excepto entre 270 y Ángulos de $315^{\circ}$.

Palabras clave: Caatinga; Meliponicultura; Anidamiento; Melipona subnitida Ducke; Frieseomelitta sp.
\end{abstract}

\title{
1. Introdução
}

As abelhas melíponas, conhecidas popularmente por abelhas nativas, abelhas sem ferrão, abelhas indígenas, abelhas tropicais ou meramente meliponíneos (Aquino, 2006, p. 26), fazem parte de um grupo composto por mais de 500 espécies (Roubik et al., 2018, p. 184). No Brasil, de acordo com dados coletados por Villas-Boas (2012), são encontradas cerca de 350 espécies. Carvalho et al. (2017) explicam que essas abelhas são caracterizadas por serem sociais, viverem em colônias permanentes e possuírem ferrão atrofiado no qual impossibilita o seu uso. Nesse grupo, as abelhas Melipona subnitida Ducke (1910) e Frieseomelitta sp., conhecidas como Jandaíra e Manoel de Abreu (Mané de Abreu), respectivamente, são largamente conhecidas por meliponicultores. Esses mesmos autores verificaram que a abelha Jandaíra, por exemplo, está presente nos nove estados do Nordeste brasileiro, possuindo caráter de endemismo em áreas localizadas ao norte do Rio Grande do Norte e na região litoral e interior do Ceará. Já a abelha Frieseomelitta possui uma maior abrangência, desde a região sudeste do país até o México (Silveira et al., 2002, p. 87).

Sobre os hábitos de nidificação dessas abelhas, Martins et al. (2004), em estudos realizados na região do Seridó no Rio Grande Norte, observaram que em 130 ninhos nidificados em árvores nativas da Caatinga, 50\% eram da abelha de $M$. subnitida Ducke, nidificadas em Commiphora leptophloeos e 22,3\% em Poincianella pyramidalis; e 5,3\% eram de abelhas Frieseomelitta doederleini Friese nidificadas em P. pyramidalis. Macedo et al. (2020), em pesquisas realizadas na região do Curimataú na Paraíba, verificaram que dos 60 ninhos de meliponínios analisados, 85\% das abelhas nativas nidificam em cavidades de árvores vivas, seguida $6,67 \%$ por árvores mortas, $5 \%$ em troncos secos e 3,33\% em cupinzeiros.

O conhecimento sobre o processo de nidificação das abelhas melíponas, de acordo com Macedo et al. (2020), vai além de uma cavidade e uma boa florada. Esses autores explicam que devem ser levados em consideração aspectos como a orientação magnética de nidificação. A busca pelo conhecimento para qual lado o orifício de entrada do ninho estará voltado tem despertado pesquisadores. Investigações sobre a orientação magnética de abelhas nativas, especialmente no bioma Caatinga, têm sido realizadas por Vaz (2015), Silva (2016), Lacerda et al. (2017), Macedo et al. (2020), Ferreira et al. (2021) e Silva et al. (2021). 
A influência do campo magnético data de 1975, quando um pesquisador da Universidade de New Hampshire em Massachusetts, EUA, demostrou que algumas bactérias aquáticas (Aquaspirillum magnetotacticum) respondem ao magnetismo terrestre, se aproximando do polo sul de uma barra magnética e se distanciando do seu polo norte, em fenômeno conhecido como magnetotactismo (Blakely, 1975). Os testes realizados com A. magnetotacticum influenciaram alguns cientistas na busca por outras descobertas sobre a influência do campo magnético da terra, levando a realização de testes com animais. Análises feitas com ressonância paramagnética eletrônica, mostram a existência de material magnético (nanopartículas) de magnetitas em formigas que podem ter influência direta nesse evento (Slowik et al., 1997; Acosta-Avalos, 2001). Esse mesmo material também foi encontrado em abdômen de abelhas Apis mellifera (Gould et al., 1978) e em abelhas sem ferrão Schwarziana quadripunctata (Lucano, 2006; Esquivel et al., 2007).

A presença de magnetita no abdômen de abelhas A. mellifera tem permitido a criação de modelos que explicam o mecanismo de orientação das abelhas. O sistema de orientação das abelhas está associado aos recursos visuais aprendidos do ambiente (Capaldi \& Dyer, 1999) para guiar seus movimentos entre o ninho e os locais de forrageamento. Além disso, o sol tem forte influência nessa dinâmica comportamental, associada com pontos de referência nos arredores do ninho que podem ser retidos na memória da abelha como um padrão distinto semelhantemente a um "relógio de sol" (Beier \& Lindauer, 1970). De igual modo, a influência magnética pode estar relacionada aos hábitos de nidificação de abelhas nativas em estudos realizados sobre a influência dos pontos cardeais e colaterais na nidificação de abelhas indígenas (Lacerda et al. 2017; Macedo et al., 2020).

Diante do exposto, o objetivo deste trabalho foi avaliar o comportamento de nidificação das abelhas nativas Melipona subnitida Ducke (1910) e Frieseomelitta sp. em seu habitat natural no Seridó oriental do estado do Rio Grande do Norte.

\section{Material e Métodos}

Esta pesquisa foi desenvolvida na Fazenda Morada da Jandaíra (FMJ), em Santana do Seridó, município localizado na microrregião do Seridó oriental do Rio Grande do Norte (Bezerra Júnior \& Silva, 2007). Adotou-se o método indutivo científico (Marconi \& Lakatos, 2009, p. 110; Pereira, 2011, p. 120) visando estimar a magnitude dos efeitos isolados ou em conjunto do comportamento de nidificação de Melipona subnitida (Ducke, 1910) e Frieseomelitta sp. As coletas de dados foram auxiliadas pelo morador da fazenda, realizando-se caminhadas em busca de ninhos nidificados em espécies vegetais nativas dessa microrregião (Figura 1). As caminhadas na busca de árvores nativas nidificadas por melíponas foram realizadas no horário de 5:00h da manhã ao meio dia, durante o período de novembro a janeiro. Cada árvore nidificada foi feita as seguintes anotações: medidas da altura do orifício de nidificação, diâmetro das árvores hospedeiras (vivas ou mortas) e posicionamento dos ninhos em relação aos pontos cardeais e colaterais. 
Figura 1. Georeferenciamento de espécies vegetais nidificadas com abelhas nativas na Fazenda Morada da Jandaíra, município de Santana do Seridó-RN.

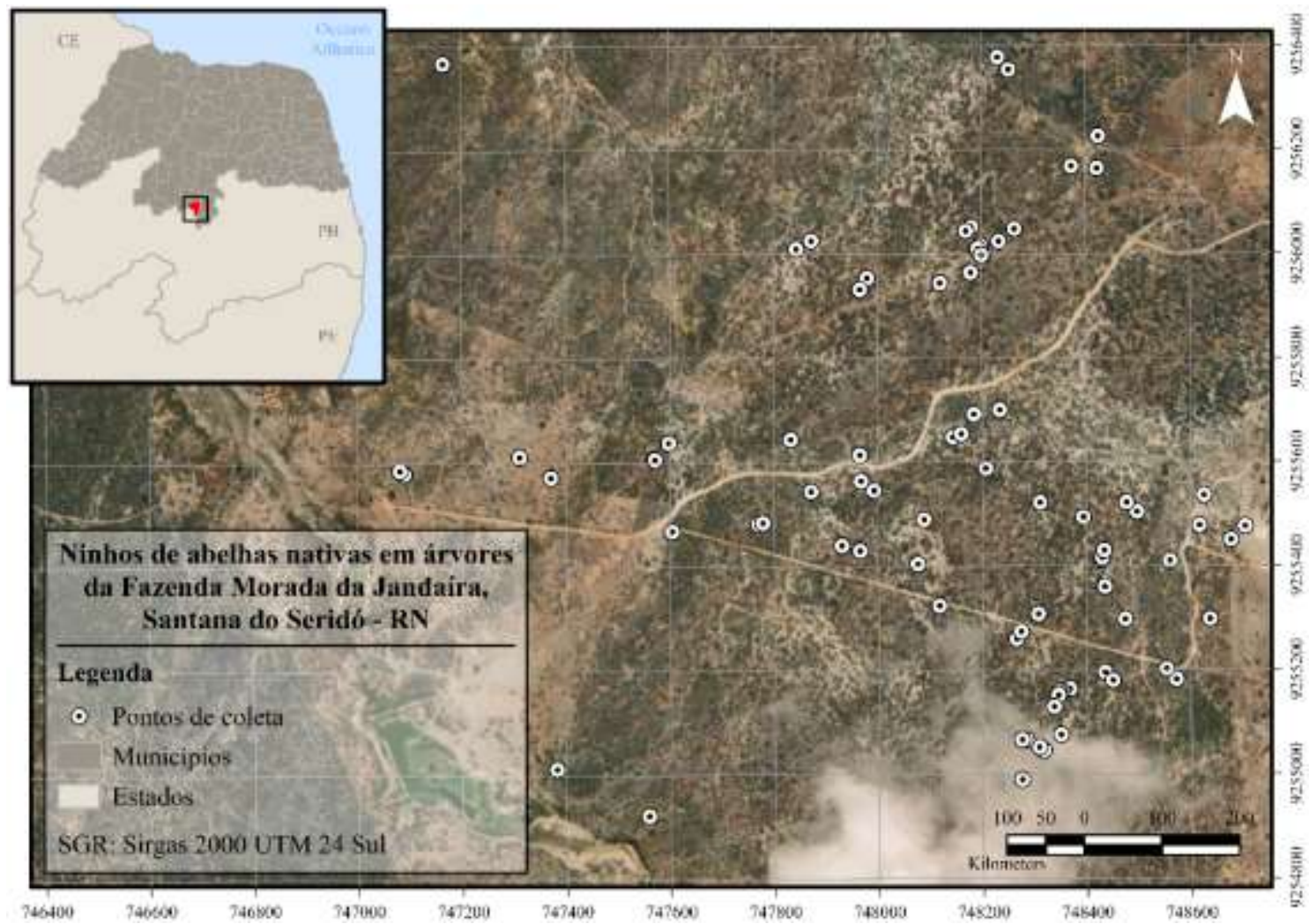

Fonte: Autores (2021).

As medidas de largura e altura dos ninhos foram feitas com o auxílio de uma fita métrica. Cada árvore nidificada recebeu uma fita de identificação para se evitar a repetição de ninhos investigados. Para a leitura (precisa) do posicionamento dos orifícios de nidificação em relação aos pontos cardeais (Norte, Sul, Leste, Oeste) e colaterais (Nordeste, Sudeste, Noroeste, Sudoeste), utilizou-se um aplicativo de bússola digital para smartphone (iPhone ${ }^{\circledR}$ Model A 1533). Os dados de velocidade do vento, umidade e temperatura foram medidos por uma mini- estação meteorológica Oregon Scientific ${ }^{\circledR}$, WMR928NX. Toda área estudada foi georeferenciada com um GPS eTrex 10 Garmin $^{\circledR}$.

\section{Análise de dados}

Inicialmente, os dados de classificação dos pontos (cardeais e colaterais) de nidificação foram transformados em dados de angulação, obedecendo a tomada de decisão de que o ponto cardeal 'Leste' representa o início da angulação circular (Zero grau) e que o ponto colateral Sudeste representa o fim da angulação circular (315 graus). A circularidade dos dados foi testada para verificar a homogeneidade dos parâmetros de concentração de múltiplas amostras de dados direcionais. Esse teste verifica se todos os grupos de interesse (espécies) obedecem a distribuição normal circular (distribuição de von Mises), sendo observada a possibilidade de aceitação da hipótese nula de que todos os grupos de interesse se encontram no mesmo parâmetro de concentração.

Para melhor visualização dos dados circulares, foram plotados histogramas do tipo polar, nos quais as informações são dadas em colunas de valores em graus $\left(0^{\circ}\right.$ a $\left.360^{\circ}\right)$. Este tipo de gráfico foi utilizado para evidenciar as orientações de nidificação relacionadas à cada espécie. Em seguida, aplicou-se uma Análise de Variância de Dados Circulares (Circular ANOVA), semelhante à análise de variância de dados lineares, com finalidade de identificar, estatisticamente, se há diferença entre os as espécies, utilizando-se as angulações de nidificação em árvores. 
As regressões Linear-Circulares foram realizadas para verificar a trajetória de preferência de orientação de nidificação em função de medidas lineares das árvores nidificadas; relação na qual a variável dependente foi a angulação da nidificação (pontos cardeais transformados) e como variável independente as variáveis de largura e altura, separadamente. As análises estatísticas foram realizadas no software R v. 2.15 (R Development Core Team, 2005) utilizando o pacote 'circular' (Agostinelli \& Lund, 2011).

\section{Resultados e Discussão}

Foram analisadas 85 árvores-hospedeiras e identificados 136 ninhos de abelhas nativas. As espécies vegetais com maior frequência de nidificação para ambas as espécies de abelhas foram umburana (Commiphora leptophloeos) e catingueira (Poincianella pyramidalis), com 69 ninhos de Melipona subnitida Ducke e 60 ninhos de Friseomelitta ssp (Tabela 1). Na Caatinga, essas árvores são referência no que diz respeito a nidificação de abelhas nativas (Martins et al., 2004). Por serem árvores importantes para o abrigo de ninhos de abelhas indígenas, Kerr (1996) alerta que muitas espécies dessas abelhas estão em perigo de extinção em decorrência do desmatamento, alterações antrópicas, uso de agrotóxico e a caça predatória de ninho pelos meleiros.

Tabela 1. Espécies de árvores analisadas, quantidade de ninhos e abelhas nativas encontradas na Fazenda Morada da Jandaíra (FMJ) Santana do Seridó-RN.

\begin{tabular}{llllc}
\hline \multicolumn{2}{c}{ Árvore hospedeira } & \multicolumn{2}{c}{ Abelha nativa } & Quantidade \\
Nome científico & Nome popular & Nome científico & Nome popular & de ninhos \\
\hline Commiphora eptophloeos & Umburana & Melipona subnitida Ducke & Jandaíra & 65 \\
Poincianella pyramidalis & Catingueira & Melipona subnitida Ducke & Jandaíra & 4 \\
Commiphora leptophloeos & Umburana & Frieseomelitta sp. & Mané de Abreu & 49 \\
Poincianella pyramidalis & Catingueira & Frieseomelitta sp. & Mané de Abreu & 11 \\
Spondias tuberosa & Umbu & Melipona subnitida Ducke & Jandaíra & 3 \\
Spondias tuberosa & Umbu & Frieseomelitta sp. & Mané de Abreu & 3 \\
Cnidoscolus quercifolius & Faveleira & Frieseomelitta sp. & Mané de Abreu & 1 \\
\hline Total & & & & $\mathbf{1 3 6}$
\end{tabular}

Fonte: Autores (2021).

A espécie C. leptophloeos, a qual obteve os maiores registros de ninhos de abelhas M. subnitida e Frieseomelitta sp. (114), possui sua grande importância no bioma Caatinga ameaçada de extinção (Kerr, 1996; Hilton-Taylor, 2000). Martins et al. (2004) explica que a retirada predatória dessas plantas leva a uma redução na oferta de substrato para a nidificação de abelhas nativas, comprometendo diretamente na reprodução dessas abelhas e, desta forma, configurando-se como uma das maiores causas de impacto negativo sobre as populações de abelhas indígenas (Thiago, 2010). Mesmo a umburana possuindo os maiores registros de ninhos nesse estudo, Martins et al. (2004) afirmam que não se pode supor uma preferência da fauna apícola por tais espécies arbóreas, uma vez que a distribuição de ninhos em espécies vegetais na Caatinga e a disponibilidade de cavidades ainda não são conhecidas. Em síntese, as abelhas nativas dependem majoritariamente da disponibilidade local de recursos alimentares (Eltz et al., 2002; Dias, 2015) em ocos de árvores vivas ou mortas (Rêgo \& Brito, 1996; Teixeira, 2001; Eltz et al., 2003) para nidificarem.

As análises de regressão circular-linear apontam que não existe associação entre os valores de altura e largura das árvores e os valores de angulação (preferência de nidificação) [Tabela 2; Figura 2; e Figura 3, a, b, c]. Porém, excetua-se a espécie 
Frieseomelitta sp. que apresentou uma relação positiva entre altura e angulação (Tabela 2; Figura 1, d); ou seja, quanto maior a altura das árvores amostradas maior nidificação nos maiores valores de angulação.

Tabela 2. Estatística descritiva das regressões circulares-lineares dos valores de angulação para os valores lineares de Largura e Altura para Melipona subnitida. e Frieseomelitta sp. t-valor - estatística t de Student, valor, p-valor - valor de probabilidade.

\begin{tabular}{lcccc}
\hline \multicolumn{1}{c}{ Largura } & \multicolumn{3}{c}{} & \\
\hline & Coeficiente & Erro Padrão & t-valor & p-valor \\
\hline Melipona subnitida & -1.326 & 2.272 & 0.584 & 0.280 \\
Frieseomelitta sp. & 2.073 & 1.642 & 1.263 & 0.103 \\
& & & & \\
\hline Altura & & & & 0.225 \\
Melipona subnitida & 0.786 & 1.040 & 0.756 & $0.013^{*}$ \\
Frieseomelitta $\mathrm{sp}$. & 0.556 & 0.251 & 2.217 &
\end{tabular}

$* \mathrm{p}<0,05-$ valor limite de associação significativa. Fonte: Autores (2021).

Sobre os valores médios de angulação para cada espécie observa-se a ANOVA circular indica diferença estatística entre as espécies (graus de liberdade = 7,54 e p < 0,001), sendo a média de 2,37 graus para as abelhas M. subnitida e de 45,68 graus para Frieseomelitta sp. (Figura 2) Esse comportamento distinto entre essas duas espécies mostra que a abelha M. subnitida é mais seletiva que a Frieseomelitta sp.

Figura 2. Dados médios de angulação, intervalos de confiança por método permutacional Bootstrap e valor de Kappa de cada espécie de abelha analisada: Melipona subnitida e Frieseomelitta sp.

\section{Melipona subnitida}

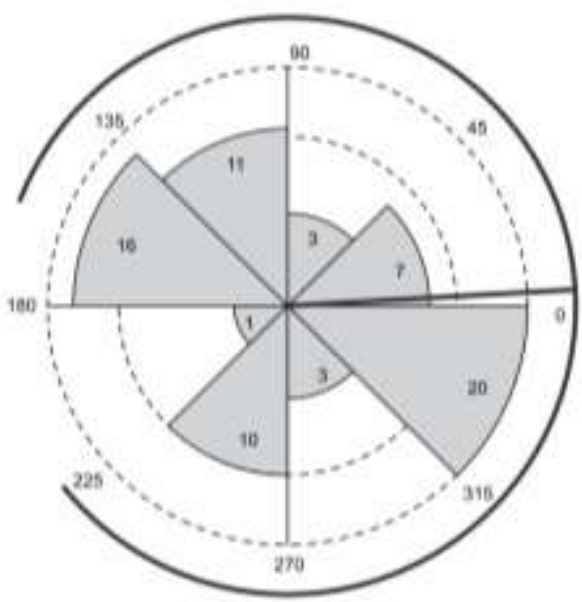

Média $=2.37$

I.C. $95 \%($ Bootstrap $)=140.9 ; 157.1$

Kappa $=0.109$
Frieseomelitta sp.

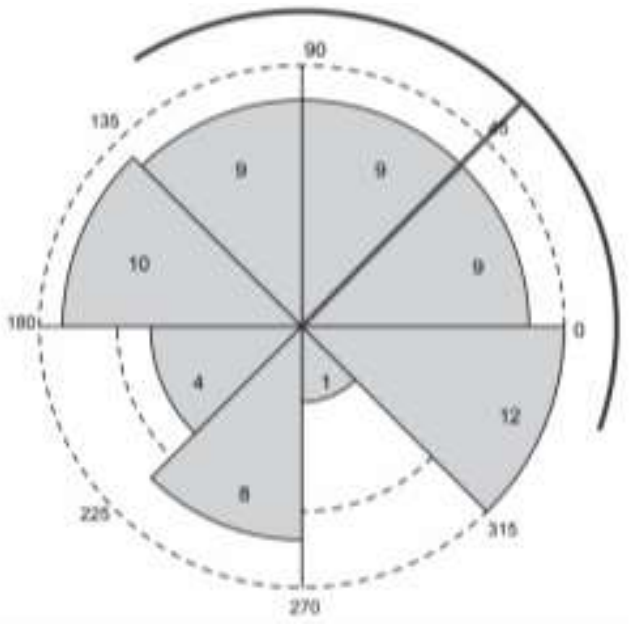

Média $=45.68$

I.C. $95 \%($ Bootstrap $)=140.9 ; 157.1$

Kappa $=0.109$

Fonte: Autores (2021). 
Em relação à escolha de orifícios de nidificação, para ambas as espécies, é prevalente para os lados com maior intensidade de sol (nascente e poente). Porém, a Frieseomelitta sp. demonstra uma maior abrangência em relação a escolha dos orifícios de nidificação. As duas espécies, entretanto, demonstram menor frequência de nidificação entre os ângulos 270 e $315^{\circ}$; angulação onde há predominância de ventos nessa área estudada. Tal comportamento assemelha-se ao número reduzido de entradas de ninho das espécies Trypoxylon aurifrons Shuckard e Centris analis (F.) em ninhos-armadilha orientadas para a direção de vento predominante (Martins et al. 2012). Essa preferência angular para nidificação é corroborada pelos achados de Frier et al. (1996), em que as abelhas demonstram que podem distinguir entre dois padrões panorâmicos de $360^{\circ}$ que são idênticos e, ainda, demonstrar que a visualização preferida nessa angulação pode ser aprendida e lembrada pelo uso oportuno nas várias sugestões direcionais.

Figura 3. Dispersão da regressão circular-linear entre os valores de angulação (transformados dos pontos cardeais) e os valores lineares de largura e altura: Melipona subnitida (a, c); Frieseomelitta sp. (b, d). Log-L - Valores da estatística de Log-Likelihood, *valor de probabilidade estatisticamente significante.
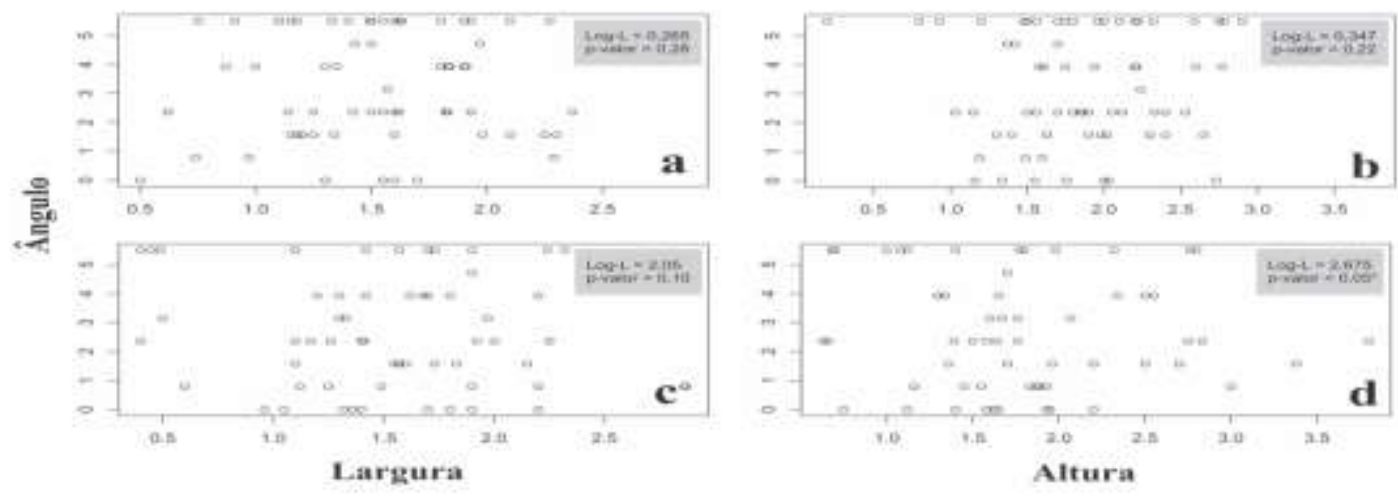

Fonte: Autores (2021).

Percebe-se, nesses resultados, que há orientação magnética que pode ser mais (ou menos) atrativa às abelhas para seu comportamento de nidificação. Esse empenho na nidificação com influência no campo magnético, com provável desenvolvimento desde o estágio pupal (Gould et al., 1978), pode ir mais além do que a "simples" preferência angular para o orifício de nidificação. Segundo Macedo et al. (2020), as abelhas nativas têm, ainda, a capacidade de alterar o diâmetro de nidificação em relação aos pontos cardeais e colaterais. Considerando que a dinâmica de ambientes em mudança e a própria diversificação existente nas microrregiões do bioma Caatinga, é provável que essas abelhas demonstrem a habilidade de aprender novos comportamentos, inclusive de nidificação, mas, também solicitar respostas reflexivas em novos contextos ambientais (Abramson, 1994, p.121).

\section{Conclusão}

As observações desse estudo permitem tirar as seguintes conclusões em relação a nidificação das abelhas Melipona subnitida Ducke: e Frieseomelitta sp. em seu habitat natural:

1. As abelhas M. subnitida Ducke e Frieseomelitta sp. nidificam com maior frequência nas espécies vegetais Commiphora leptophloeos e Poincianella pyramidalis;

2. A abelha M. subnitida Ducke demonstra maior frequência de nidificação na direção Noroeste (NW); e

3. A abelha Frieseomelitta sp. possui uma frequência de nidificação em qualquer orientação magnética, exceto entre os ângulos 270 e $315^{\circ}$. 
Estudos adicionais são necessários para se conhecer o hábito de nidificação das abelhas $M$. subnitida Ducke e Frieseomelitta sp. em outras microrregiões do bioma Caatinga.

\section{Agradecimentos}

Os autores expressam seus agradecimentos aos proprietários da Fazenda Morada da Jandaíra, por disponibilizarem sua fazenda a realização desta pesquisa; Vanderli e família pelo acolhimento e auxílio nas trilhas realizadas na propriedade; Andreia Vasconcellos, pela coleta de dados em campo; meliponicultor Ezequiel Roberto de Medeiros Macedo, pela assistência técnica durante os estudos; Bruna Candeia pela arte georeferenciamento (mapa) das espécies nidificadas nessa pesquisa; e a Coordenação de Aperfeiçoamento de Pessoal de Nível Superior (CAPES), pela concessão de bolsa de estudos (pós-graduação) ao primeiro autor.

\section{Referências}

Abramson, C. I. (1994). A prime of invertebrate learning: the behavioral perspective. Washington: American Psychological Assocition, 273 p.

Acosta-Avalos, D., Esquivel, D. M. S., Wajnberg, H. G. P., Lins de Barros, P. S. \& Oliveira, I. L. (2001). Seasonal patterns in the orientation system of the migratory ant Pachycondyla marginate. Naturwissenschaften, 88(1): 343-346. 10.1007/s001140100245.

Agostinelli, C. \& Lund, U. (2011). R package 'circular': circular statistics (version 0.4-3). https://r-forge.r-project.org/projects/circular/.

Aquino, I. S. (2006). Abelhas nativas da Paraíba. Editora Universitária/UFPB, 91p.

Beier, W. \& Lindauer, M. (1970). Der sonnenstand als zeitgeber für die biene. Apidologie, 1(1): 5-28. https://hal.archives-ouvertes.fr/hal-00890278/document.

Bezerra Júnior, J. G. O. \& Silva, N. M. (2007). Caracterização geoambiental da microrregião do seridó oriental do rio grande do norte. Holos, 23(2): 78-91. https://doi.org/10.15628/holos.2007.102

Blakely, R. J. (1975). Magnetotatic bactéria. Science, 190(4212): 378-379. http://web.csulb.edu/ cwallis/170/text/Blakemore.Magnetotactic.Bacteria.1975.pdf.

Carvalho, A. T., Maia-Silva, C., Jaffé, R., Souza, B. A., Zanella, F. C. V., Martins, C. F., Carvalho, C. A. L., Alves, R. M. O., Koedam, D., Acosta, A. L., Imperatriz-Fonseca, V. L. \& Giannini, T. C. (2017). Distribuição geográfica atual da abelha jandaíra e previsões para a sua distribuição futura. In: ImperatrizFonseca, V. L., Koedam, D. \& Hrncir, M. (Ed.). A abelha jandaíra no passado, no presente e no futuro. Mossoró: EDUFERSA, 73-78. https://edufersa.ufersa.edu.br/wp-content/uploads/sites/27/2017/10/abelha-jandai\%CC\%81ra-livro-eletronico.pdf

Capaldi, E. A. \& Dyer, F. C. (1999). The role orientation flights on homing performance in honey bees. Journal of Experimental Biology, 202: $1655-1666$. https://doi.org/10.1242/jeb.202.12.1655

Dias, A. B. (2015). Ninhos de abelhas nativas sem ferrão (Meliponineae) em ambiente urbano. [trabalho de conclusão de curso]. Botucatu: Universidade Estadual Paulista, Instituto de Biociências de Botucatu. https://repositorio.unesp.br/bitstream/handle/11449/142880/000866983.pdf?sequence=1\&isAllowed=y.

Eltz, T., Brühl, C. A., Kaars, S. V. D. \& Linsenmair, K. E. (2002). Determinants of stingless bee nest density in lowland dipterocarp forests of Sabah, Malaysia. Oecologia, 131(1): 27-34. http://doi.org/10.1007/s00442-001-0848-6.

Eltz, T., C.A. Brühl, Z. Imiyabir, K.E. Linsenmair. (2003). Nesting and nest trees of stingless bees (Apidae: Meliponini) in lowland dipterocarp forests in Sabah, Malaysia, with implications for forest management. Forest Ecology and Management, 172(1): 301-313. http://doi.org/10.1016/s0378-1127(01)00792-7.

Esquivel, D. M. S., Wajnberg, E., Nascimento, F. S., Pinho, M. B., Lins-de-Barros, G. G. P., Eizemberg, R. (2007). Do geomagnetic storms change the behaviour of the stingless bee guiruçu (Schwarziana quadripunctata)? Die Naturwissenschaften. 94. 139-42. 10.1007/s00114-006-0169-z

Ferreira, R. C. C., Aquino, I. S., Vital, A. F. M., Silva, A. B. \& Barbosa, A. S. (2021). Preliminary observations on the nesting of the cupira bee (Partamona cupira Smith) in the Caatinga biome. Research, Society and Development, 10(7): e58610716956. 10.33448/rsd-v10i7.16956.

Frier, H., Edwards, E., Smith, C. Neale, S., Collett, T. (1996). Magnetic compass cues and visual pattern learning in honey bees. Journal of Experimental Biology, 199(6): 1353-1361. https://doi.org/10.1242/jeb.199.6.1353

Gould, J. L., Kirschvink, J. L. \& Deffeyes, K. S. (1978). Bees have magnetic remanence. Science, 201(4360): 1026-1028.

10.1126/science.201.4360.1026

Kerr, W. E., Carvalho, G. A. \& Nascimento, V. A. (1996). Abelha urucu: biologia, manejo e conservação. Coleção Manejo da vida silvestre 2. Acangaú, 144 p.

Marconi, M. A. \& Lakatos, E. M. (2009). Metodologia do trabalho científico: procedimentos básicos; pesquisa bibliográfica, projeto e relatório; publicações e trabalhos científicos. (7a ed.), Atlas, 225 p.

Lacerda, D. C. O., Aquino, I. S. Borges, P. F. \& Barbosa, A. S. (2017). Influência dos pontos cardeais e colaterais na nidificação de abelhas nativas em colmeias octogonais. Gaia Scientia, 11(2): 203-217. http://dx.doi.org/10.21707/gs.v11.n02a014 
Lucano, M. J. (2006). Caracterização de material magnético mineralizado em abelhas meliponini Schwarziana quadripunctata: magnetometria SQUID e Ressonância Ferromagnética. Tese de doutorado: Centro Brasileiro de Pesquisas Físicas. http://livros01.livrosgratis.com.br/cp058256.pdf.

Macedo, C. R. C., Aquino, I. S., Borges, P. F., Barbosa, A. S. \& Medeiros, G. R. (2020). Comportamento da nidificação de abelhas melíponas. Ciência Animal Brasileira, 21(1). https://www.revistas.ufg.br/vet/article/view/58736

Martins, C. F., Ferreira, R. P. \& Carneiro, L. T. (2012). Influence of the orientation of nest entrance, shading, and substrate on sampling trap-nesting bees and wasps. Neotropical Entomology, 41(2): 105-111. 10.1007/s13744-012-0020-5.

Martins, C. F., Laurino, M. C., Koedam, D. \& Imperatriz-Fonseca, V. L. (2004). Espécies arbóreas utilizadas para nidificação por abelhas sem ferrão na caatinga (Seridó, PB; João Câmara, RN). Biota Neotropica, 4(2): 1-8. https://www.scielo.br/j/bn/a/v8WJDkQJcSk5xwPjs5JCwJd/?lang=pt\&format=pdf.

Pereira, M. G. (2011). Artigos científicos: como redigir, publicar e avaliar. Koogan, 383 p.

R Development Core Team (2005). R: a language and environment for statistical computing, R Foundation for Statistical Computing. www.rproject.org.

Rêgo, M. M. C. \& Brito. (1996). Abelhas sociais (Apidae: Meliponini) em um ecossistema de cerrado s.l. (Chapadinha - MA, BR): distribuição dos ninhos. In: Encontro sobre abelhas, $3^{\circ}$, Ribeirão Preto, Anais..., Ribeirão Preto, FFCLRP-USP, 3(1):238-247. http://www.scielo.br/scielo.php?script=sci_nlinks\& ref=000137\&pid=S0073-4721200900010000200036\&lng=en.

Roubik, D. W. (2018). Applied pollination and selected studies. In: Roubik, D. W. The pollination of cultivated plants: A compendium for practitioners. Vol 1, chapter 9: 183-197. Italy, Rome: Food and Agriculture Organization of the United Nations, 324 p. https://ainfo.cnptia.embrapa.br/digital/bitstream/item/180143/1/I9201EN-1.pdf.

Silva, F. J. A. (2016). Preferência de nidificação em abelhas indígenas. Dissertação de Mestrado em Ciências Agrárias (Agroecologia, Universidade Federal da Paraíba-UFPB, 90 p.

Silva, F. J. A., Aquino, I. S., Barbosa, A. S. \& Borges, P. F. (2021). Nesting behavior of Melipona scutellaris (Latreille, 1811). Research, Society and Development, 10(7): e7310716350. 10.33448/rsd-v10i7.16350.

Silveira, F. A., Melo, G. A. R \& Almeida, E. A. B. (2002). Abelhas brasileiras, sistemática e identificação. Belo Horizonte: Fernando A. Silveira, 253 p.

Slowick, T. J., Green, B. L. \& Thorvilson, H. G. (1997). Detection of magnetism in the red imported fire ant (Solenopsis invicta) using magnetic resonance imaging. Bioeletromagnetics, 18(5): 396-399. https://www.semanticscholar.org/paper/Detection-of-magnetism-in-the-red-imported-fire-an-SlowikGreen/fb04d1fe42fe2f107cc25024c76bd6988ccf2f5a.

Teixeira, A. F. R. (2001). Abelhas indígenas (Hymenoptera: Meliponini) residentes em uma área de caatinga na margem esquerda do Rio São Francisco, Ibiraba, Barra, Bahia. Monografia (Bacharelado em Ciências Biológicas) - Instituto de Biologia, Universidade Federal da Bahia, Salvador, BA. 42 p.

Thiago, P. S. S. (2010). Ecologia da nidificação de Mandaçaia-do-chão (M. quinquefasciata) no Parque Estadual Veredas do Peruaçu, MG. Biota, Belo Horizonte, 3(2): 24-37.

Vaz, M. A. (2015). Influência dos pontos cardeais e colaterais na nidificação de abelhas nativas. Dissertação de Mestrado, Programa de Pós-Graduação em Ciências Agrárias (Agroecologia), Universidade Federal da Paraíba, 33 p.

Villas-Bôas, J. (2012). Manual Tecnológico: Mel de abelhas sem ferrão. Brasília: Instituto Sociedade, População e Natureza (ISPN). Brasil, 96 p. 